\title{
Quantum ballistic transport in strained epitaxial germanium
}

Y. Gul, S. N. Holmes, P. J. Newton, D. J. P. Ellis, C. Morrison, M. Pepper, C. H. W. Barnes, and M. Myronov

Citation: Appl. Phys. Lett. 111, 233512 (2017);

View online: https://doi.org/10.1063/1.5008969

View Table of Contents: http://aip.scitation.org/toc/apl/111/23

Published by the American Institute of Physics

\section{Articles you may be interested in}

Fabrication and characterization of a germanium nanowire light emitting diode

Applied Physics Letters 111, 233103 (2017); 10.1063/1.5006152

Magnetic two-dimensional field effect transistor

Applied Physics Letters 111, 233508 (2017); 10.1063/1.4994634

Gate tunable parallel double quantum dots in InAs double-nanowire devices

Applied Physics Letters 111, 233513 (2017); 10.1063/1.4997646

Temperature-modulated electronic structure of graphene on SiC: Possible roles of electron-electron interaction and strain

Applied Physics Letters 111, 231603 (2017); 10.1063/1.4986425

Charge carrier velocity in graphene field-effect transistors

Applied Physics Letters 111, 233505 (2017); 10.1063/1.5003684

Separating grain-boundary and bulk recombination with time-resolved photoluminescence microscopy Applied Physics Letters 111, 233902 (2017); 10.1063/1.5010931

\section{Scilight} Sharp, quick summaries illuminating the latest physics research

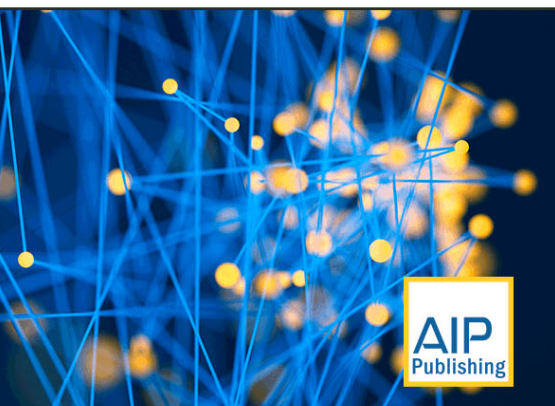




\title{
Quantum ballistic transport in strained epitaxial germanium
}

\author{
Y. Gul, ${ }^{1, a)}$ S. N. Holmes, ${ }^{2}$ P. J. Newton, ${ }^{3}$ D. J. P. Ellis, ${ }^{2}$ C. Morrison, ${ }^{4}$ M. Pepper, ${ }^{1}$ \\ C. H. W. Barnes, ${ }^{3}$ and M. Myronov ${ }^{4}$ \\ ${ }^{1}$ London Centre for Nanotechnology, University College London, 17-19 Gordon Street, London WC1H 0AH, \\ United Kingdom \\ ${ }^{2}$ Toshiba Research Europe Limited, Cambridge Research Laboratory, 208 Cambridge Science Park, \\ Milton Road, Cambridge CB4 OGZ, United Kingdom \\ ${ }^{3}$ Department of Physics, Cavendish Laboratory, University of Cambridge, J. J. Thomson Avenue, \\ Cambridge CB3 OHE, United Kingdom \\ ${ }^{4}$ Department of Physics, University of Warwick, Coventry CV4 7AL, United Kingdom
}

(Received 11 October 2017; accepted 26 November 2017; published online 7 December 2017)

\begin{abstract}
Large scale fabrication using Complementary Metal Oxide Semiconductor compatible technology of semiconductor nanostructures that operate on the principles of quantum transport is an exciting possibility now due to the recent development of ultra-high mobility hole gases in epitaxial germanium grown on standard silicon substrates. We present here a ballistic transport study of patterned surface gates on strained Ge quantum wells with SiGe barriers, which confirms the quantum characteristics of the Ge heavy hole valence band structure in 1-dimension. Quantised conductance at multiples of $2 \mathrm{e}^{2} / \mathrm{h}$ is a universal feature of hole transport in Ge up to $10 \times\left(2 \mathrm{e}^{2} / \mathrm{h}\right)$. The behaviour of ballistic plateaus with finite source-drain bias and applied magnetic field is elucidated. In addition, a reordering of the ground state is observed. Published by AIP Publishing.

https://doi.org/10.1063/1.5008969
\end{abstract}

CMOS (Complementary Metal Oxide Semiconductor) compatible nanostructures in germanium have developed rapidly ${ }^{1}$ but without the observation of ballistic effects that are characterised by quantisation of the electrical conductance. ${ }^{2,3}$ This has been partly due to material quality; however, recent improvements in $\mathrm{SiGe}$ growth technology 4,5 with reduced background impurity levels and the introduction of thin Ge quantum well layers into SiGe barriers have achieved hole mobilities $(\mu)$ of $\sim 4500 \mathrm{~cm}^{2} / \mathrm{V} \mathrm{s}$ at ambient temperature and $\sim 10^{6} \mathrm{~cm}^{2} / \mathrm{V} \mathrm{s}$ at low temperature. Earlier mobilities in this material were orders of magnitude smaller. ${ }^{6}$ The improvement in mobility has led to the clear ballistic conductance quantisation reported here in narrow quantum wells of p-type Ge. The International Technology Roadmap for Semiconductors (ITRS) ${ }^{7}$ has identified $\mathrm{p}-\mathrm{Ge}$ as a candidate for the channel replacement material in p-MOS, and the ballistic transport presented here not only strengthens the case for Ge inclusion in the ITRS but also demonstrates that $\mathrm{p}-\mathrm{Ge}$ has device potential for nanoelectronic and quantum computing applications. Recent publications have also outlined the application of $\mathrm{p}-\mathrm{Ge}$ for Majorana fermion studies. $^{8,9}$

The nominally undoped Ge system with $\mathrm{Si}_{0.3} \mathrm{Ge}_{0.7}$ barriers studied in this work is a very low defect, epitaxial material. This feature limits the influence of dopants and defects on the electrostatics of the quantum confinement, provides low leakage surface gating with dielectric layers of $\mathrm{SiO}_{2}$, and gives a long mean free path in the Ge channel sufficient for largely defect-free, ballistic conductance features to be quantified. In general, quantised conductance in a ballistic patterned gate device is observed in the differential conductance $(G)$ at values $g_{v} g_{s} N^{2} / h$, where $g_{s}$ is the spin

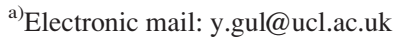

degeneracy, $\mathrm{g}_{\mathrm{v}}$ is the valley degeneracy, $\mathrm{N}$ is the (integer) quantum number of one-dimensional channels ${ }^{2,3}$ defined between the gates, and $\mathrm{e}$ and $\mathrm{h}$ are the unit of charge and Planck's constant, respectively.

In n-type strained $\mathrm{Si} / \mathrm{SiGe}$ etched narrow channels and quantum point contacts, a valley degeneracy $\left(\mathrm{g}_{\mathrm{v}}\right)$ of 2 was observed $^{10,11}$ with quantised conductance plateaus separated by $2 \mathrm{G}_{0}$, with $\mathrm{G}_{0}=2 \mathrm{e}^{2} / \mathrm{h}$. This valley degeneracy could be removed by strong in-plane confinement ${ }^{12}$ with plateaus then separated by $\mathrm{G}_{0}$. The valley splitting can be engineered to be greater than the spin splitting. ${ }^{13}$ In contrast to the conduction band, the heavy hole $(\mathrm{HH})$ valence band in Ge has only a single valley at the $\Gamma$ point with $g_{v}=1$. In self-assembled $p-G e$ core, Si-cladding nanowires, a weak ballistic feature was identified among classical Coulomb Blockade features, and with suppressed back scattering in this particular device, ballistic effects were observed at high temperatures. ${ }^{14}$ Although there has been no experimental report of quantised conductance in $\mathrm{p}-\mathrm{Ge} / \mathrm{SiGe}$ wafers, the conductance of the one-dimensional hole gas has been predicted to be partially quantised depending on the energy $\Delta$, light, and heavy hole splitting. ${ }^{15}$ This model predicts a modified ballistic conductance staircase dependent on the exchange interaction being either ferromagnetic or anti-ferromagnetic with just a single plateau at $0.5 \mathrm{G}_{0}$ for large $\Delta(>10 \mathrm{meV})$, irrespective of the sign of the exchange interaction. This is the experimental situation investigated here where $\Delta$ is calculated to be $\sim 94 \mathrm{meV}$.

The band structure of the $11 \mathrm{~nm}$ thick Ge is modified from bulk Ge by the compressive biaxial strain with the $\mathrm{Si}_{0.3} \mathrm{Ge}_{0.7}$ barrier cladding, see Fig. 1(d). The ground state wavefunction of the two dimensional hole gas (2DHG) is from the heavy hole $(\mathrm{HH})$ band, with total angular momentum (J) 3/2 aligned along the out-of-plane strain axis with components $\left(\mathrm{J}_{\mathrm{Z}}\right) \pm 3 / 2$ and a carrier effective mass $\left(\mathrm{m}^{*}\right) \sim 0.065 \mathrm{~m}_{\mathrm{e}}$, 


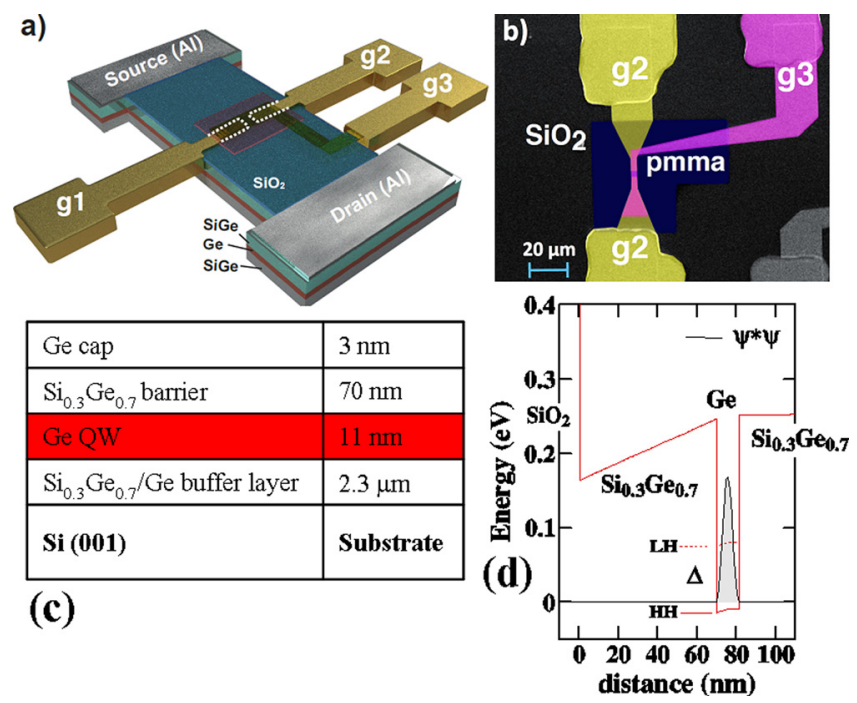

FIG. 1. (a) The overall device scheme. The patterned gates g1 and g2 define the 1-dimensional channel, and the global top gate g3 controls the carrier density in the channel. (b) An SEM image of a patterned gate region showing the pmma electron beam resist, top gate dielectric underneath the g3 metallisation. (c) A cross-section of the Ge quantum well and barrier layers used for the device sets A, B, and C. (d) A self-consistent calculation of the confining potential and the charge distribution. $\Delta$ is the light hole to heavy hole energy splitting.

where $\mathrm{m}_{\mathrm{e}}$ is the free electron mass. ${ }^{16,17}$ The light hole (LH) states $\left(\mathbf{J}=3 / 2\right.$ and $\left.\mathbf{J}_{\mathrm{z}}= \pm 1 / 2\right)$ in the Ge valence band are higher in energy by $\sim 94 \mathrm{meV}$ from the heavy hole state, so any applied current is only carried by heavy hole states, irrespective of the measurement temperature or applied gate voltages. The hole gas is confined to the Ge quantum well region as shown in Fig. 1(d), where a self-consistent wavefunction $\Psi * \Psi$ and potential is plotted. The compressive strain is fundamentally important in the p-Ge system as it provides the quantisation axis for $\mathbf{J}$ along the growth direction perpendicular to the conducting plane. In these devices, there is no Rashba-type spin splitting in the two dimensional contact regions as $\mathbf{J} \cdot \mathbf{k}_{\|}=0$ and $\Delta \mathrm{E}_{\text {Rashba }} \sim\left(\mathbf{J} \times \mathbf{k}_{\|}\right) \cdot \mathbf{E}_{\mathbf{z}} \approx 0$, where $\mathbf{E}_{\mathbf{z}}$ is the electric field in the growth direction and $\mathbf{k}_{\|}$is the linear momentum vector in the plane of the 2DHG contact regions (with components $\mathrm{k}_{\mathrm{x}}$ and $\mathrm{k}_{\mathrm{y}}$ ). In the 2DHG, only a Zeeman spin splitting is observed ${ }^{18}$ for large $\Delta$. A cubic $\left(\mathbf{k}_{\|}{ }^{3}\right)$ dependent Rashba effect has been previously identified ${ }^{19-21}$ in p-Ge, but this effect is too small to be observed in the wafers measured here due to the low carrier density and large compressive strain. The patterned gates (g1 and g2) control the width of the 1-D channel and the global top gate (g3) controls the carrier density in the channel, see Fig. 1(a). Figure 1(b) shows a scanning electron microscopy (SEM) image of the surface gating arrangement. A persistent photoconductivity effect is used to generate $\sim 1 \times 10^{11} \mathrm{~cm}^{-2}$ holes in the device.

The experimental dependence of the ballistic transport in $\mathrm{p}$-Ge with top gate voltage $\left(\mathrm{V}_{\mathrm{g} 3}\right)$, in-plane magnetic field, differential channel definition voltage (where $\mathrm{V}_{\mathrm{g} 1} \neq \mathrm{V}_{\mathrm{g} 2}$ ), and finite source-drain dc voltage is reported in this work. Three different sets of devices (A, B, and C) were fabricated and measured with the transport data on device set $\mathrm{C}$ presented here. Devices B and C had a global top gate to allow the carrier density to be changed in the 1-D channel, and this is shown schematically in Fig. 1(a). The differential conductance as a function of patterned gate voltage at $20 \mathrm{mK}$ in device $\mathrm{C}$ is shown in Fig. 2(a). The top gate is stepped from $0 \mathrm{~V}$ on the right hand side of the figure (where $\mathrm{p} \sim 1 \times 10^{11}$ $\mathrm{cm}^{-2}$ ) to $+1 \mathrm{~V}$ (depletion with $\mathrm{p} \sim 0$ in the channel). Reproducible and clearly defined conductance plateaus are seen at up to $7 \mathrm{G}_{0}$, in the depletion mode with $\mathrm{V}_{\mathrm{g} 3}>0$. The plateau separation is consistent with spin degenerate, single valley conduction as expected. At conductance $<\mathrm{G}_{0}$, a shoulder at $\sim 0.7 \mathrm{G}_{0}$ is seen in $\mathrm{p}-\mathrm{Ge}$. This feature is characteristic of ballistic 1-dimensional channels ${ }^{22,23}$ and eventually weakens here at higher top gate voltages. The disorder is evident as peaks or shoulders in the depletion mode just before the channel pinches-off. Re-illuminating the device can remove this structure, indicating that the origin is in disorder.

The in-plane g-factor for the $\mathrm{p}$-Ge heavy hole gas is $\sim 0$ as the angular momentum $\mathbf{J}$ is pinned in the growth direction (out of plane) as discussed above [see Ref. 24]. However, a large enough in-plane magnetic field (B) can remove this degeneracy in the angular momentum $\mathrm{J}_{\mathrm{z}}= \pm 3 / 2$ states, and at $\sim 2 \mathrm{~T}$, the half-plateaus start to appear from 0.5 to $4.5 \mathrm{G}_{0}$, see Fig. 2(b), which shows in-plane fields to 6T. This is further evidence that the plateaus are ballistic with the hole gas showing $g_{v}=1$ and $g_{s}=1$ in Fig. 2(b) under those conditions. The half-plateaus appear due to a Zeeman splitting, albeit with a small g-factor, rather than due to a spin-orbit coupling effect that has been seen in InGaAs based materials. ${ }^{25-28}$ We note from Fig. 2(a) that at a low carrier concentration, small plateaus appear at low values of conductance, and these will be discussed elsewhere.
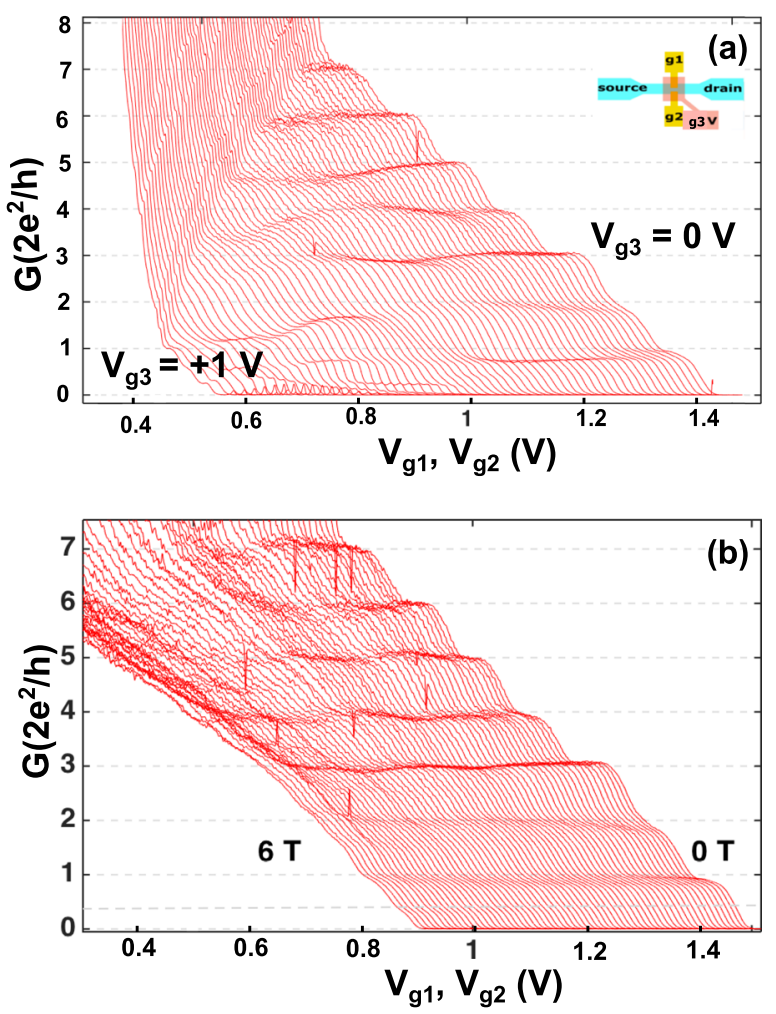

FIG. 2. (a) The differential conductance as a function of patterned gate voltage at $20 \mathrm{mK}$. The global top gate voltage is stepped in $+5 \mathrm{mV}$ increments from 0 to $1 \mathrm{~V}$ (on the extreme left). (b) The differential conductance as a function of patterned gate voltage with $\mathrm{B}$ in-plane from 0 to $6 \mathrm{~T}$ in steps of $0.1 \mathrm{~T}$. The top gate voltage $\mathrm{V}_{\mathrm{g} 3}$ was at $0 \mathrm{~V}$. 
Figure 3(a) shows device $\mathrm{C}$ in the enhancement mode, a negative voltage on the global gate g3. The top gate voltage is stepped from $0 \mathrm{~V}$ on the left hand side to $-0.2 \mathrm{~V}$ on the right hand side in increments of $-10 \mathrm{mV}$. In the enhancement mode, conductance plateaus up to $10 \mathrm{G}_{0}$ can be resolved, demonstrating that a clean ballistic channel has been fabricated.

At $\mathrm{V}_{\mathrm{g} 3}=0$, the left hand trace of Fig. 3(a), an asymmetric bias $\left(\Delta \mathrm{V}=\mathrm{V}_{\mathrm{g} 1}-\mathrm{V}_{\mathrm{g} 3}\right)$ to gates $\mathrm{g} 1$ and $\mathrm{g} 2$ was applied and is shown in Fig. 3(b). This has the effect of laterally shifting the 1-dimensional channel between the surface gates g1 and g2. As Fig. 3(b) shows, the integer conductance plateaus are robust and insensitive to the lateral shift. However, additional features do occur such as the $0.7 \mathrm{G}_{0}$ structure as discussed earlier. For some combinations of $\mathrm{V}_{\mathrm{g} 1}$ and $\mathrm{V}_{\mathrm{g} 2}$, double jumps can be seen in the conductance. This could be a manifestation of a re-ordering of the ground state into double rows of charge ${ }^{29}$ with $\mathrm{G}$ appearing as $2 \mathrm{G}$. This scenario is likely to be observed in high mobility structures and is a further good indication of the quality of the p-Ge hole gases reported in this work. This is the first such observation of the strong many body interactions in hole gases and is being investigated further. A lateral spin-orbit coupling effect ${ }^{26,27}$ is not observed for asymmetric biasing of gates $\mathrm{g} 1$ and $\mathrm{g} 2$.

Applying a dc voltage bias between the source and drain contacts of the device removes the momentum degeneracy for the two physical directions of current flow along the channel. Figures 4(a) and 4(b) show the conductance of
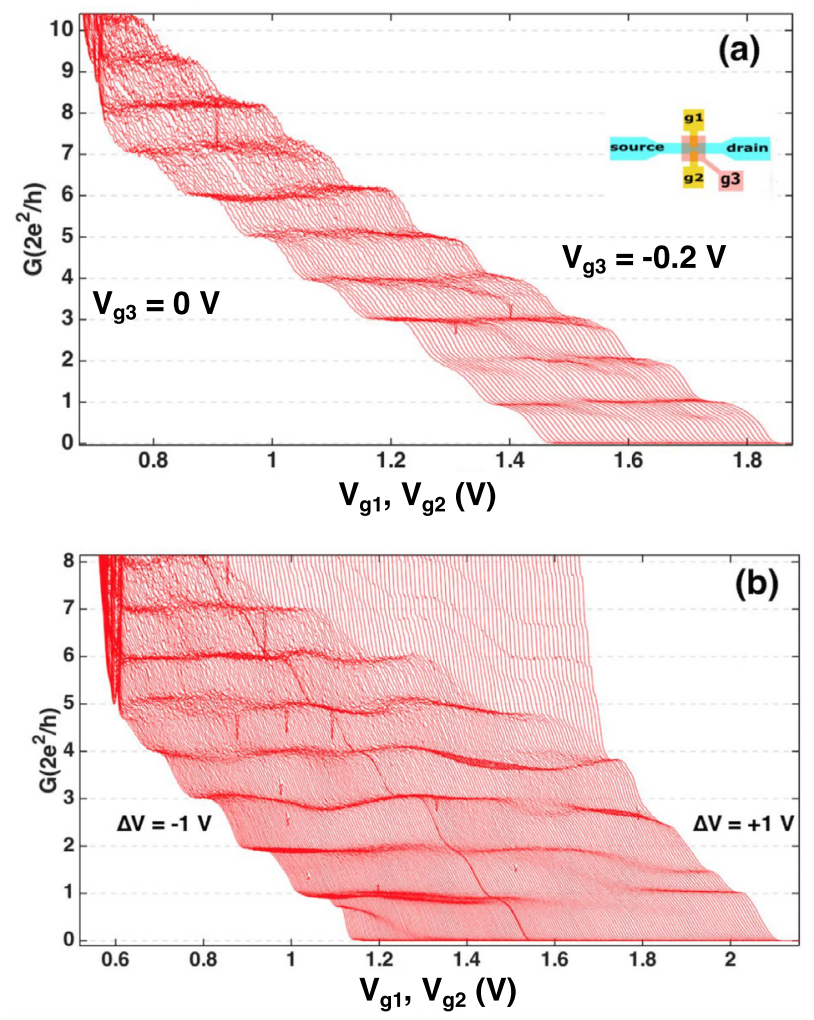

FIG. 3. (a) The differential conductance as a function of pattered gate voltage at $20 \mathrm{mK}$ in the enhancement mode. The global top gate voltage is stepped in $-10 \mathrm{mV}$ increments from 0 to $-0.2 \mathrm{~V}$ (extreme right trace). (b) Asymmetric bias (with $\mathrm{V}_{\mathrm{g} 1} \neq \mathrm{V}_{\mathrm{g} 2}$ ) to the patterned gates up to $\mathrm{V}_{\mathrm{g}_{1}}-\mathrm{V}_{\mathrm{g} 2}$ (labelled as $\Delta \mathrm{V}$ ) $= \pm 1 \mathrm{~V}$ at $20 \mathrm{mK}$ in increments of $\Delta \mathrm{V}=10 \mathrm{mV}$. For all traces, $\mathrm{V}_{\mathrm{g} 3}=0 \mathrm{~V}$. The thick line pinching off at $1.54 \mathrm{~V}$ is the case for $\Delta \mathrm{V}=0$.
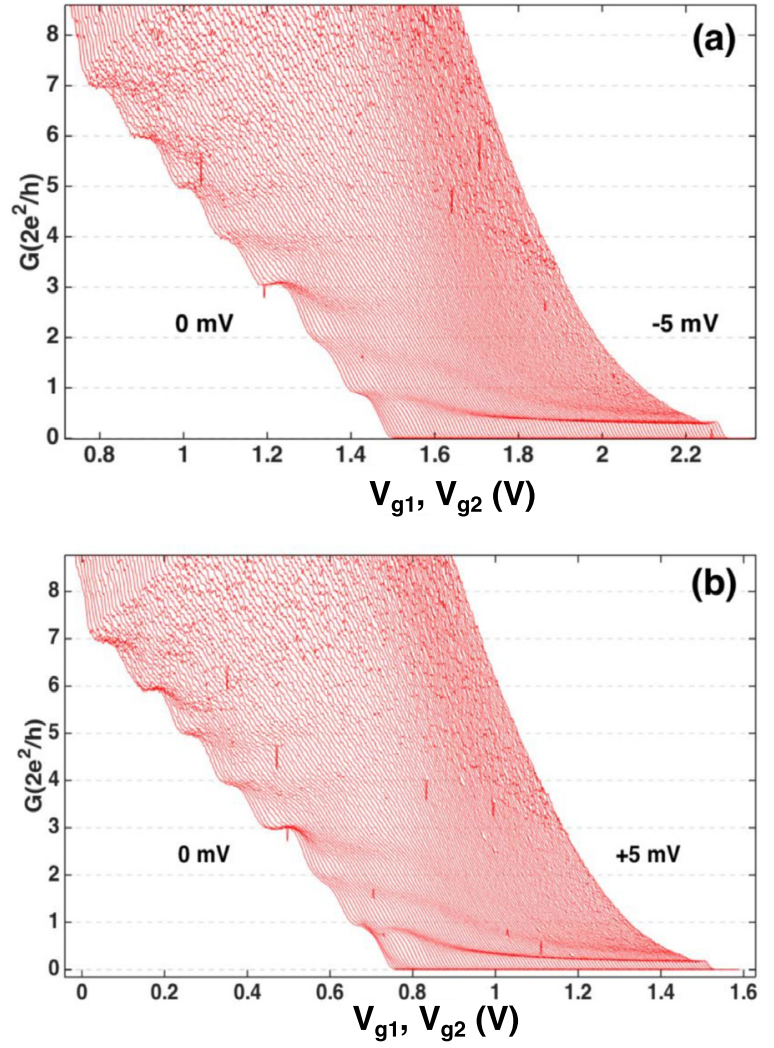

FIG. 4. The differential conductance as a function of patterned gate voltage at $20 \mathrm{mK}$ with $\mathrm{V}_{\mathrm{g} 3}=0 \mathrm{~V}$. In (a), a dc source-drain bias is added from 0 to $-5 \mathrm{mV}$ in steps of $-50 \mu \mathrm{V}$. In (b), a dc source-drain bias is added from 0 to $5 \mathrm{mV}$ in steps of $+50 \mu \mathrm{V}$.

device $\mathrm{C}$ as a function of the patterned gate voltages $\mathrm{V}_{\mathrm{g} 1}$ and $\mathrm{V}_{\mathrm{g} 2}$ with an additional dc voltage that is incremented from 0 to $-5 \mathrm{mV}$ and $+5 \mathrm{mV}$, respectively. The two figures are symmetric with dc voltage as expected from the starting point (on the left hand side of the figures) showing integer plateaus up $7 \mathrm{G}_{0}$. The integer plateaus have gone by $\sim \pm 0.7 \mathrm{mV}$, the 1-dimensional subband separation, and are replaced by $1 / 2$-integer plateaus. At $\mathrm{G}<\mathrm{G}_{0}$, clear plateaus have developed at $0.5 \mathrm{G}_{0}$ and $0.25 \mathrm{G}_{0}$ with finite source drain voltage. The plateau at $0.5 \mathrm{G}_{0}$ is the spin resolved plateau where the spin-degeneracy has been removed without an applied magnetic field. The $0.25 \mathrm{G}_{0}$ plateau has been seen in a GaAs channels and has been identified ${ }^{30}$ as a fully spinpolarised uni-directional current. A slight difference in the value of the final plateau with the direction of voltage indicates a slight asymmetry in the voltage drop at the ends of the device as the average value is 0.25 .

The observation of heavy hole spin splitting in the ballistic conductance of p-Ge was predicted in Ref. 15 and is confirmed here up to $10 \mathrm{G}_{0}$. This letter has laid the ground work for understanding the heavy hole spin splitting in p-Ge in 1-dimension considering that the angular momentum vector $\mathbf{J}$ is confined along the growth direction. Applied sourcedrain voltages and asymmetric gating of the channel have uncovered plateaus at half integer values as well as a ballistic structure down to $0.25 \mathrm{G}_{0}$. The quality of epitaxial germanium is now sufficiently high that it can compete with GaAs-based devices that operate on the principles of ballistic transport. This system can be further studied, and the role 
played by the compressive strain and control of the light hole contribution can be understood in Ge for p-MOS, fundamental spintronic devices, and Majorana fermion studies.

The work programme at the University of Cambridge and Warwick University was funded by the EPSRC, "Spintronic device physics in Si/Ge heterostructures," EP/ J003263/1 and a platform Grant No. EP/J001074/1. Y. Gul thanks Toshiba Research Europe Limited for the provision of a CASE award studentship and Dr. S. Kumar for assistance with the Triton fridge system at University College London.

${ }^{1}$ R. Pillarisetty, Nature 479, 324 (2011).

${ }^{2}$ D. A. Wharam, T. J. Thornton, R. Newbury, M. Pepper, H. Ahmed, J. E. Frost, D. G. Hasko, D. C. Peacock, D. A. Ritchie, and G. A. C. Jones, J. Phys. C 21, L209 (1988).

${ }^{3}$ B. J. van Wees, H. van Houten, C. W. J. Beenaker, J. G. Williamson, L. P. Kouwenhoven, D. van der Marel, and C. T. Foxon, Phys. Rev. Lett. 60, 848 (1988).

${ }^{4}$ M. Myronov, C. Morrison, J. Halpin, S. Rhead, C. Casteleiro, J. Foronda, V. A. Shah, and D. Leadley, Jpn. J. Appl. Phys., Part 1 53, 04EH02 (2014).

${ }^{5}$ M. Myronov, C. Morrison, J. Halpin, S. Rhead, J. Foronda, and D. Leadley, Solid State Electron. 110, 35 (2015).

${ }^{6}$ F. Schäffler, Semicond. Sci. Technol. 12, 1515 (1997).

${ }^{7}$ See http://www.itrs2.net/ for "International Technology Roadmap for Semiconductors (ITRS)," 2016.

${ }^{8}$ J. Xiang, A. Vidan, M. Tinkham, R. M. Westervelt, and C. M. Lieber, Nat. Nanotechnol. 1, 208 (2006).

${ }^{9}$ F. Maier, J. Klinovaja, and D. Loss, Phys. Rev. B 90, 195421 (2014).

${ }^{10}$ U. Wieser, U. Kunze, K. Ismail, and J. O. Chu, Appl. Phys. Lett. 81, 1726 (2002).

${ }^{11}$ J. F. von Pock, D. Salloch, G. Qiao, U. Wieser, T. Hackbarth, and U. Kunze, J. Appl. Phys. 119, 134306 (2016).

${ }^{12}$ G. Frucci, L. Di Gaspare, F. Evangelisti, E. Giovine, A. Notargiacomo, V. Piazza, and F. Beltram, Phys. Rev. B 81, 195311 (2010).
${ }^{13}$ S. Goswami, K. A. Slinker, M. Friesen, L. M. McGuire, J. L. Truitt, C. Tahan, L. J. Klein, J. O. Chu, P. M. Mooney, D. W. van der Weide et al., Nat. Phys. 3, 41 (2007).

${ }^{14}$ W. Lu, J. Xiang, B. P. Timko, Y. Wu, and C. M. Lieber, Proc. Natl. Acad. Sci. 102, 10046 (2005).

${ }^{15}$ M. Rosenau da Costa, I. A. Shelykh, and N. T. Bagraev, Phys. Rev. B 76, 201302(R) (2007).

${ }^{16}$ I. B. Berkutov, V. V. Andrievskii, Yu. F. Komnik, O. A. Mironov, M. Mironov, and D. R. Leadley, Low Temp. Phys. 35, 141 (2009).

${ }^{17}$ M. Failla, M. Myronov, C. Morrison, D. R. Leadley, and J. Lloyd-Hughes, Phys. Rev. B 92, 045303 (2015).

${ }^{18}$ S. N. Holmes, P. J. Newton, J. Llandro, R. Mansell, C. H. W. Barnes, C. Morrison, and M. Myronov, J. Appl. Phys. 120, 085702 (2016).

${ }^{19}$ R. Moriya, K. Sawano, Y. Hoshi, S. Masubuchi, Y. Shiraki, A. Wild, C. Neumann, G. Abstreiter, D. Bougeard, T. Koga et al., Phys. Rev. Lett. 113, 086601 (2014).

${ }^{20}$ C. Morrison, P. Wisniewski, S. D. Rhead, J. Foronda, D. R. Leadley, and M. Myronov, Appl. Phys. Lett. 105, 182401 (2014).

${ }^{21}$ J. Foronda, C. Morrison, J. E. Halpin, S. D. Rhead, and M. Myronov, J. Phys.: Condens. Matter 27, 022201 (2015).

${ }^{22}$ K. J. Thomas, J. T. Nichols, M. Y. Simmons, M. Pepper, D. R. Mace, and D. A. Ritchie, Phys. Rev. Lett. 77, 135 (1996).

${ }^{23}$ L. W. Smith, H. Al-Taie, F. Sfigakis, P. See, A. A. J. Lesage, B. Xu, J. P. Griffiths, H. E. Beere, G. A. C. Jones, D. A. Ritchie et al., Phys. Rev. B 90, 045426 (2014).

${ }^{24}$ R. Winkler, D. Culcer, S. J. Papadakis, B. Habib, and M. Shayegan, Semicond. Sci. Technol. 23, 114017 (2008).

${ }^{25}$ P. J. Simmonds, F. Sfigakis, H. E. Beere, D. A. Ritchie, M. Pepper, D. Anderson, and G. A. C. Jones, Appl. Phys. Lett. 92, 152108 (2008).

${ }^{26}$ P. Debray, S. M. S. Rahman, J. Wan, R. S. Newrock, M. Cahay, A. T. Ngo, S. E. Ulloa, S. T. Herbert, M. Muhammad, and M. Johnson, Nat. Nanotechnol. 4, 759-764 (2009).

${ }^{27}$ J. Wan, M. Cahay, P. Debray, and R. Newrock, Phys. Rev. B 80, 155440 (2009).

${ }^{28}$ N. Bhandari, M. Dutta, J. Charles, R. S. Newrock, M. Cahay, and S. T. Herbert, Adv. Nat. Sci.: Nanosci. Nanotechnol. 4, 013002 (2013).

${ }^{29}$ S. Kumar, K. J. Thomas, L. W. Smith, M. Pepper, G. L. Creeth, I. Farrer, D. Ritchie, G. Jones, and J. Griffiths, Phys. Rev. B 90, 201304(R) (2014).

${ }^{30}$ T.-M. Chen, A. C. Graham, M. Pepper, I. Farrer, and D. A. Ritchie, Appl. Phys. Lett. 90, 032102 (2008). 